Línea de Investigación

Salud y Calidad de Vida,

Escuela de Psicología,

Universidad Católica del

Norte. Antofagasta, Chile.

aPsicólogo, Magíster en

Salud Pública, Doctor en

Psicología Clínica y de la

Salud

bPsicólogo, Licenciado en Psicología.

Este artículo es producto de un estudio financiado por la Comisión Nacional de Investigación Científica y Tecnológica del Gobierno de Chile-CONICYT, a través del proyecto FONDECYT 11100090

Recibido el 8 de mayo de 2012, aceptado el 4 de septiembre de 2012.

Correspondencia a: Dr. Alfonso Urzúa. Escuela de Psicología, Universidad Católica del Norte. Avenida Angamos 0610. Antofagasta, Chile.

E-mail: alurzua@ucn.cl

\section{Calidad de vida en adultos mayores: análisis factoriales de las versiones abreviadas del WHOQoL-Old en población chilena}

\author{
ALFONSO URZÚA M. ${ }^{\mathrm{a}}$, MAURICIO NAVARRETE $^{\mathrm{b}}$
}

\section{Factor analysis of abbreviated versions of the WHOQoL-Old in Chilean older people}

\begin{abstract}
Background: The increase in elderly population demands the need for new health measures that are reliable and valid. Aim: To compare the factor structure of the Quality of Life Scale WHOQoL-Old of the World Health Organization with the three proposed abbreviated versions of the scale. Material and Methods: We performed confirmatory factor analysis on data collected from 804 older people, aged $70 \pm 6$ years (66\% females). Results: One of the unifactorial models that incorporate six questions had goodness of fit values greater than 0.95 in the non-normed fit index and below .05 in the root-mean square error of approximation. Conclusions: The abbreviated version tested is a valid and reliable questionnaire to be used in Chilean older people.

(Rev Med Chile 2013; 141: 28-33).
\end{abstract}

Key words: Aged: Evaluation; Quality of Life; Studies as topic.
E n los últimos años, la población adulta mayor (AM) se ha incrementado de manera considerable, principalmente debido al mejoramiento de las condiciones de vida ${ }^{1} \mathrm{y}$ al aumento de la expectativa de ésta². En Chile, se estima que la población de AM al 2020 será de 3,2 millones de habitantes, esto es, un AM por cada cinco personas ${ }^{3}$.

Resultados de estudios que se han realizado en esta población dan cuenta, entre otros, de la evaluación de sus condiciones de vida y cómo en esta impacta su estado de salud ${ }^{4}$, siendo posibles de aplicar en la creación de políticas públicas ${ }^{5}$, el diseño de programas centrados en la promoción de un envejecimiento activo y saludable ${ }^{6}$ o la generación de proyectos en salud, orientados tanto a la prevención como en la educación del $\mathrm{AM}^{2,7,8}$.

Ante este aumento poblacional, se hace necesario realizar estudios que no sólo estén centrados en la morbilidad, los factores de riegos y la prevalencia de enfermedades crónicas en personas AM, sino orientadas a la calidad del envejecimiento y al bienestar psicológico del $\mathrm{AM}^{9,10}$, en otras palabras, un énfasis en la disminución de la situación de vulnerabilidad a la que se ven expuestos ${ }^{8}$ tal como el impacto que vivencia el AM en su calidad de vida (CV) producto de los eventos que se desarrollan en el proceso de envejecimiento ${ }^{11}$. La CV según la Organización Mundial de la Salud (OMS) puede ser definida como "la percepción individual de la posición en la vida en el contexto de la cultura y sistema de valores en el cual se vive y su relación con las metas, expectativas, estándares e intereses"12. En términos operativos, puede entenderse como el nivel percibido de bienestar derivado de la evaluación que realiza cada persona de elementos objetivos y subjetivos en distintas dimensiones de su vida ${ }^{13}$.

Los estudios en CV que se han realizado en Chile en la población AM se han focalizado en la influencia de la vivienda ${ }^{14}$, en el análisis de las percepciones, creencias y evaluaciones de la $\mathrm{CV}$ en personas semivalentes ${ }^{15}$, en el comportamiento psicométrico de escalas generales de evaluación de $\mathrm{CV}$ en esta población específica ${ }^{16} \mathrm{y}$ en describir los 
factores vinculados en la CV en esta población ${ }^{7}$. Dos de estas investigaciones utilizaron el cuestionario general de CV WHOQoL-Bref $1^{14-16}$, una un cuestionario específico para $\mathrm{AM}^{7}$ y la última metodología cualitativa recolectando información a través de entrevistas en profundidad ${ }^{15}$.

La utilización de instrumentos específicos para la medición de la CV en la población AM favorece una evaluación integral de las diversas dimensiones que condicionan el bienestar del adulto mayor $^{11}$, dado que permite obtener información específica sobre la vivencia de la persona en esa etapa de su vida, tal como la escala WHOQoL-Old, instrumento internacionalmente empleado en la evaluación de la calidad de vida (CV) enfocado principalmente en la población AM. Este instrumento, pese a que ha reportado buenas propiedades psicométricas tanto en su versión original ${ }^{17}$, como en adaptaciones a otros idiomas ${ }^{18-20}$, puede ser demasiado extenso, principalmente en adultos de mayor edad, por lo que se han propuesto versiones más cortas de este instrumento ${ }^{21}$.

En Chile, pese a que se ha comenzado a utilizar el WHOQoL-Old con fines de investigación, no se ha reportado estudios sobre las propiedades psicométricas en nuestra población adulto mayor.

Esta investigación tuvo por objetivo comparar la estructura factorial propuesta en la versión original del WHOQoL-Old ${ }^{17}$ con las versiones abreviadas propuesta por Fang et al, de dicha escala ${ }^{21}$ en población de adultos mayores chilenos.

\section{Material y Método}

\section{Participantes}

Dada las dificultades de obtener una muestra representativa, se realizó un muestreo intencio- nado por disponibilidad en el cual participaron 804 personas: 528 mujeres $(65,7 \%)$ y 276 hombres $(34,3 \%)$. El rango de edad de los encuestados fue de 60 a 90 años. La edad promedio de la muestra total fue de 70,17 años ( $\mathrm{DE}=6,551)$, siendo para las mujeres de 70,43 años $(\mathrm{DE}=6,70)$ y la de los hombres de 69,69 años ( $\mathrm{DE}=6,246)$.

Los criterios de exclusión para participar en la investigación fueron la presencia evidente de deterioro físico o mental o de estados avanzados de deterioro cognitivo (demencia o Alzheimer en fase inicial), que impidieran responder los cuestionarios. Los cuestionarios fueron administrados por uno de los autores del trabajo, apoyado por miembros del grupo de neuropsicología de la Escuela de Psicología de la Universidad Católica del Norte, quienes están entrenados para aplicar cuestionarios y aplicar los criterios de inclusión y exclusión requeridos en cada investigación.

\section{Instrumento}

WHOQoL-Old. Su origen se remonta a los cuestionarios WHOQoL-100 o el WHOQoL-Bref que fueron utilizados para medir la CV en la población adulta más joven, de los cuales se pensaba que podrían ser de uso en la población AM. Estimando la posibilidad de que ciertas dimensiones son valoradas de una perspectiva propia en los distintos grupos etarios, se realizaron una serie de estudios pilotos en diversas regiones como Inglaterra, España, Francia, Japón, Brasil y otros que dieron como resultado la culminación de este instrumento transcultural ${ }^{17}$.

Este cuestionario está conformado por un total de 24 ítems de escala Likert con 5 opciones cada una y 6 dimensiones preponderantes (Tabla 1). El puntaje de las 6 facetas proporciona un puntaje

Tabla 1. Dominios de calidad de vida evaluados por el WHOQoL-Old

\begin{tabular}{|c|c|}
\hline Dominio & Definición \\
\hline Habilidades sensoriales & Impedimento sensorial que afecta la vida diaria y el funcionamiento general \\
\hline Autonomía & $\begin{array}{l}\text { Capacidad para tomar decisiones propias, sentirse en control del propio futuro y de hacer las } \\
\text { cosas que se quiere }\end{array}$ \\
\hline $\begin{array}{l}\text { Actividades del pasado, } \\
\text { presente y futuras }\end{array}$ & Grado de satisfacción con los logros pasados y futuros y con el reconocimiento de lo realizado \\
\hline Participación social & Grado de satisfacción en las distintas actividades que participa \\
\hline Muerte y el morir & Grado de preocupación hacia la muerte y el morir \\
\hline Intimidad & Oportunidad para amar y ser amado \\
\hline
\end{tabular}


global que nos permite tener un acercamiento inicial sobre la CV en AM. Se han reportado propiedades psicométricas adecuadas y consistentes tanto en población americana con la creación de tres versiones reducidas de la WHOQoL-Old con una consistencia interna de $0,681,0,678$ y $0,649^{21}$. De la misma forma, en España se han realizado estudios para comprobación de la idoneidad del instrumento, reportándose propiedades psicométricas adecuadas y consistentes con un Alfa de Cronbach $0,80^{19}$. Por último, en la población brasileña se reporta un Alfa de Cronbach 0,71 a $0,88^{18}$ y en la población chilena con un Alfa de Cronbach 0,837. El WHOQoL-Old constituye un módulo específico para adultos mayores que complementa a las versiones del WHOQoL-100 y WHOQoL-Bref, debido a que incluye aspectos específicos relacionados con la experiencia del envejecimiento.

\section{Procedimientos}

Este reporte forma parte de un proyecto de investigación en calidad de vida en población chilena, el cual fue aprobado por los Comités de ética de la Universidad Católica del Norte y de la Comisión Nacional de Investigación Científica y Tecnológica-CONICYT. El cuestionario fue aplicado a 884 adultos mayores, pertenecientes a diferentes servicios gubernamentales como no gubernamentales y en lugares públicos de la ciudad. Los cuestionarios fueron mayormente aplicados por los evaluadores y un número menor aplicado grupalmente. En ambas modalidades aplicación tuvieron un promedio de duración de 45 min. Del número total de cuestionarios aplicados, regresaron 804 completados de manera satisfactoria. Previamente a la aplicación del instrumento a cada participante se le explicó en qué consistía este instrumento, los fines que tenía y las respectivas instrucciones para completar el cuestionario. A los que aceptaban participar, se les solicitó que firmaran un consentimiento escrito. Una vez recolectados los cuestionarios, estos fueron ingresados a una base de datos creada con el software SPSS 17.

\section{Análisis Estadístico}

Se realizaron análisis descriptivos de las variables como el análisis de confiabilidad del instrumento mediante el estadístico Alfa de Cronbach. Los análisis factoriales confirmatorios fueron realizados utilizando el programa EQS 6.1 para Windows.

\section{Resultados}

\section{Confiabilidad}

Para evaluar la confiabilidad, se analizó la consistencia interna de los datos mediante el estadístico Alfa de Cronbach.

El alfa para la escala total considerando los 24 ítems fue de 0,80 , en tanto para el modelo 1 fue de 0,53 , para el modelo 2 de 0,46 y para el modelo 3 fue de 0,50.

La Tabla 2 muestra las correlaciones ítem test menos

Tabla 2. Correlación ítem factor modelos evaluados

\begin{tabular}{|c|c|c|c|c|}
\hline$\stackrel{n}{\text { pregunta }}$ & $\begin{array}{c}\text { Modelo } \\
06 \text { factores }\end{array}$ & $\begin{array}{c}\text { Modelo } \\
A^{i}\end{array}$ & $\begin{array}{c}\text { Modelo } \\
\text { B }^{\mathrm{ii}}\end{array}$ & $\begin{array}{c}\text { Modelo } \\
\text { Ciii }^{\text {in }}\end{array}$ \\
\hline 1 & 0,46 & & & \\
\hline 2 & 0,53 & & 0,08 & \\
\hline 3 & 0,53 & & & \\
\hline 4 & 0,55 & & & 0,26 \\
\hline 5 & 0,48 & & & \\
\hline 6 & 0,66 & $-0,04$ & 0,08 & $-0,06$ \\
\hline 7 & 0,75 & & & \\
\hline 8 & 0,73 & & & \\
\hline 9 & 0,52 & & & \\
\hline 10 & 0,47 & & & \\
\hline 11 & 0,32 & 0,46 & 0,41 & \\
\hline 12 & 0,47 & 0,48 & 0,41 & \\
\hline 13 & 0,47 & & & \\
\hline 14 & 0,39 & & & \\
\hline 15 & 0,49 & & & \\
\hline 16 & 0,66 & 0,36 & & \\
\hline 17 & 0,71 & & 0,26 & 0,41 \\
\hline 18 & 0,54 & & & \\
\hline 19 & 0,45 & & & 0,43 \\
\hline 20 & $-0,41$ & 0,26 & & 0,31 \\
\hline 21 & 0,58 & 0,33 & & \\
\hline 22 & 0,65 & & 0,29 & \\
\hline 22 & 0,71 & & & \\
\hline 24 & 0,738 & & & 0,32 \\
\hline
\end{tabular}

'preguntas $06,11,12,16,20,21$ de la versión original; iipreguntas $02,06,11,12,17,22$ de la versión original; iiipreguntas 04, 06, 17, 19, 20, 24 de la versión original. 
Tabla 3. Estadísticos de Bondad de Ajuste distintos modelos

\begin{tabular}{|c|c|c|c|c|c|c|c|c|c|}
\hline Modelo & $\chi^{2}$ & $\chi^{2} / g l$ & CFI & NFI & GFI & AGFI & RMRS & & RMSEA \\
\hline 06 factores & 895,301 & $895,301 / 237=3,77$ & 0,90 & 0,87 & 0,919 & 0,88 & 0,07 & 0,06 & $(0,056-0,064)$ \\
\hline 01 factor $A^{i}$ & 62,193 & $62,193 / 9=6,91$ & 0,91 & 0,90 & 0,97 & 0,94 & 0,07 & 0,09 & $(0,066-0,107)$ \\
\hline 01 factor $B^{i i}$ & 83,109 & $83,109 / 9=9,23$ & 0,87 & 0,86 & 0,97 & 0,92 & 0,08 & 0,10 & $(0,082-0,122)$ \\
\hline 01 factor Ciii & 9,983 & $9,83 / 9=1,03$ & 1,00 & 0,98 & 1,00 & 1,00 & 0,02 & 0,012 & $(0,000-0,043)$ \\
\hline
\end{tabular}

ipreguntas 06, 11, 12,16, 20, 21 de la versión original; "iipreguntas 02, 06, 11, 12, 17, 22 de la versión original; iiipreguntas 04, 06, 17, 19, 20, 24 de la versión original. CFI= Comparative Fix Index; NFI= Normed Fit Index; GFI= Goodness of Fit Index; $\mathrm{AGFI}=$ Adjusted Goodness of Fit Index; RMRS = Standardized Root Mean Square Residual; RMSEA= Root Mean Square Error of Approximation.

el ítem. Tal como se puede apreciar, en los modelos abreviados evaluados, las mejores correlaciones se encuentran en la propuesta C, con excepción de la pregunta seis, que no presenta correlación con el factor.

\section{Estructura factorial}

La Tabla 3 nos muestra los estadísticos de bondad de ajuste, considerando el modelo teórico de seis factores relacionados y los tres modelos unifactoriales propuestos por Fang et $\mathrm{al}^{21}$. El valor de $\chi^{2}$ fue significativo para todos los modelos evaluados $(\mathrm{p}<, 01)$.

En general, se observa un ajuste moderado de los valores a los modelos evaluados. Los valores que mejor se ajustan a lo esperado en la razón $\chi^{2} /$ grados de libertad (se espera que esta razón sea menor de 3), sólo son posibles de encontrar en el modelo original de 6 factores y en la propuesta $\mathrm{C}$ de tres factores, siendo este único modelo que tiene un valor bajo 3. Se espera que los indicadores de ajuste CFI, NFI, GFI y AGFI, sean mayores e iguales a $0,95^{22}$, valor que es alcanzado en todos estos sólo en el modelo C.

En el indicador RMRS, el cual se espera sea próximo a cero, el valor menor y, por ende expresión de un mejor ajuste de los datos, se encuentra nuevamente en el modelo C. Similar hecho se reproduce con los valores del indicador RMSEA (se espera sean menores a 0,08 ), en donde los únicos modelos que se ajustan a este valor son el original de seis factores y el Modelo C.

\section{Discusión}

La utilización de medidas de salud centradas en el bienestar de los adultos mayores puede constituir un importante insumo no tan sólo en la práctica clínica, sino también en mediciones de salud poblacional. En este contexto, la confiabilidad y validez son imprescindibles al momento de seleccionar un instrumento de evaluación. En este marco, esta investigación tuvo por objeto comparar los indicadores de bondad de ajuste de la propuesta original de seis factores de la Organización Mundial de la Salud, con las tres versiones abreviadas propuestas por Fang et $\mathrm{al}^{21}$, dado a que como este autor plantea, la práctica indica que uno de los principales problemas que tenemos con algunos instrumentos específicos para el adulto mayor, como el caso del WHOQoL-Old, es su extensión, especialmente para AM con dificultades de visión, discapacidad física o enfermedades graves.

Los resultados permiten concluir que la propuesta unifactorial C de seis preguntas (anexo 1), constituye el único modelo que presenta un ajuste aceptable en tanto indicadores de bondad de ajuste $^{22}$. Si bien la correlación ítem-factor es baja y el valor del Alfa de Cronbach es moderado, esto es esperable dado el número de ítems que compone el factor. Una de las preguntas del modelo amerita una mayor revisión, siendo la que inquiere sobre el grado de preocupación por la muerte. Posiblemente, el problema con este reactivo sea a que el término "preocuparse", a nivel coloquial en Chile, puede tener una doble significación, ya sea en términos positivos (prestar atención) o bien en términos negativos, por lo que sería necesario mejorar este ítem.

Una limitación que debe convertirse en una futura demanda de investigación, es su aplicación en distintas regiones del país, ya que la muestra es exclusivamente de una ciudad, por lo que es necesario investigar su comportamiento con muestras nacionales, siendo esta una primera aproximación. 
La relevancia de esta primer acercamiento dice relación con que la utilización de un cuestionario más breve, pese a que implica la pérdida de información detallada por dominios, puede constituirse en una importante herramienta para la práctica clínica cotidiana, al evaluar resultados de tratamientos, la evaluación de un paciente o simplemente para medir su estado de salud percibida, sino también como una medida de tamizaje en salud para mediciones a nivel poblacional.

\section{Anexo 1. Propuesta unifactorial C}

\begin{tabular}{|r|l|}
\hline $\mathbf{n}^{*}$ & Pregunta \\
\hline 4 & ¿En qué medida siente que tiene control sobre su futuro? \\
\hline 6 & ¿Qué tan preocupado/a está sobre la manera en que morirá? \\
\hline 17 & ¿En qué grado está satisfecho/ con su nivel de actividad? \\
\hline 19 & Si mira su vida hacia atrás. ¿Qué tan contento/a está con las cosas que recuerda? \\
\hline 20 & ¿Cómo evaluaría el funcionamiento de sus sentidos (por ejemplo, audición, visión, gusto, olfato, tacto)? \\
\hline 24 & ¿En qué medida tiene oportunidades para ser amado/a? \\
\hline
\end{tabular}

*Número de pregunta en versión original.

\section{Referencias}

1. Hernández Z. Variables condicionantes en la calidad de vida de personas de tercera edad. Revista de Psicología Iberoamericana 1997; 2: 23-32.

2. Peláez M. La construcción de las bases de la buena salud en la vejez: situación en las Américas. Rev Panam Salud Publica/Pan Am J Public Health 2005; 17 (5/6): 299-302.

3. Instituto Nacional de Estadística de Chile. INE. Enfoque estadístico: Población adulta mayor en el bicentenario. Gobierno de Chile, Instituto Nacional de Estadística de Chile. Santiago, Chile. 2010.

4. Sánchez H, Albala C. Desigualdades en salud: adulto en comunas del Gran Santiago. Rev Med Chile 2004; 132 (4): 453-60.

5. Wong R, Espinoza M, Palloni A. Adultos mayores mexicanos en contexto socioeconómico amplio: salud y envejecimiento. Salud Pública México 2010: 49 (4); 436-47.

6. Menéndez J, Guevara A, Arcia N, León EM, Marín C, Alfonso JC. Enfermedades crónicas y limitación funcional en adultos mayores: estudio comparativo en siete ciudades de América Latina y el Caribe. Rev Panam Salud Pública 2005; 17 (5/6) 353-61.

7. Urzúa A, Bravo M, Ogalde M, Vargas C. Factores vinculados a la calidad de vida en la adultez mayor. Rev Med Chile 2011; 139 (8): 1006-14.

8. Jürschik P, Escobar MA, Nuin C, Botigué T. Criterios de fragilidad del adulto mayor. Estudio piloto. Atención Primaria 2011: 43 (4) 190-6.
9. Mella R, González L, D’Appolonio J, Maldonado I, Fuenzalida A, Díaz A. Factores Asociados al Bienestar Subjetivo en el Adulto Mayor. Psykhe 2004; 13 (1): 7989.

10. Ortiz J, Castro M. Bienestar psicológico de los adultos mayores, su relación con la autoestima y la autoeficacia: Contribución de enfermería. Cienc. Enferm 2009; 15 (1): 25-31.

11. Cortés AM, Villarreal E, Galicia L, Martínez L, Vargas ER. Evaluación geriátrica integral del adulto mayor. Rev Med Chile 2011; 139 (6): 725-31.

12. WHOQoL GROUP. The World Health Organization Quality of life assessment (WHOQoL). Position Paper from the World Health Organization. Social Science \& Medicine 1995; 41 (10): 1403-09.

13. Urzúa A, Caqueo-Urízar A. Calidad de vida: una revisión teórica del concepto. Terapia Psicológica 2012; 30: 61-71.

14. Torres H, Quezada M, Rioseco R, Ducci ME. Calidad de vida de adultos mayores pobres de viviendas básicas: Estudio comparativo mediante uso de WHOQoLBREF. Rev Med Chile 2008; 136 (3): 325-33.

15. Osorio P, Torrejón MJ, Meersohn C, Anigstein MS. Comprensión de la Calidad de Vida en personas semivalentes en Chile. Salud \& Sociedad 2011; 2 (2): 203-17.

16. Espinoza I, Osorio P, Torrejón MJ, Lucas-Carrasco R, Bunout D. Validación del cuestionario de calidad de vida (WHOQoL-BREF) en adultos mayores chilenos. Rev Med Chile 2011; 139: 579-86. 
Estructura Factorial del WHOQoL-Old en población chilena - A. Urzúa et al

17. Power M, Quinn K, Schmidt S, WHOQoL-OLD group. Development of the WHOQOL-Old module. Qual Life Res 2005; 14: 2197-214.

18. Fleck M. Development and validation of the Portuguese version of the WHOQoL-Old module. Rev Saúde Pública 2006; 40 (5): 785-91.

19. Carrasco R, Laidlaw K, Power MJ. Suitability of the WHOQoL-Bref and WHOQoL-Old for Spanish older adults. Aging Ment Health 2011; 15 (5): 595-604.

20. Chachamovich E, Fleck M, Trentini C, Power M. Bra- zilian WHOQoL-Old Module version: a Rasch analysis of a new instrument. Rev Saúde Pública 2008; 42 (2): 308-16.

21. Fang J, Power M, Lin Y, Zhang J, Hao Y, Chatterji S. Development of Short Versions for the WHOQoL-Old Module. The Gerontologist 2011; 52 (1): 66-78.

22. Batista JM, Coenders G, Alonso J. Análisis factorial confirmatorio. Su utilidad en la validación de cuestionarios relacionados con la salud. Med Clin (Barc) 2004; 122: 21-7. 